\title{
NOVEL OPTICAL DIRECT DETECTION SCHEME FOR DPSK SIGNALS USING FIBRE BRAGG GRATINGS
}

\author{
P. Munoz ${ }^{1}$, I. T. Monroy ${ }^{2}$, R. Garcia ${ }^{1}$, J.J. Vegas ${ }^{2}$, F.M. Huijskens ${ }^{2}$, S. Sales ${ }^{1}$, \\ A. Gonzalez ${ }^{1}$, J. Capmany ${ }^{1}$, A.M.J. Koonen ${ }^{2}$ \\ ${ }^{1}$ IMCO2, Universidad Politecnica de Valencia, Camino de Vera s/n, 46022 Valencia - SPAIN - \\ ssales@dcom.upv.es \\ ${ }^{2}$ COBRA Institute, Eindhoven University of Technology, The Netherlands, I.Tafur@tue.nl
}

\begin{abstract}
A novel scheme for direct detection of DPSK signals using FBGs is proposed. It alleviates the stability requirements of conventional one-bit-delay demodulators and it is suitable for very high data-rates.
\end{abstract}

\section{INTRODUCTION}

Differential Phase-Shift Keying (DPSK) has been proposed as an attractive alternative to On-Off Keying (OOK) in optical fibre communication systems since it is robust to the nonlinear transmission impairments [1,2]. Balanced DPSK receivers using a one-bit-delay interferometer have been widely proposed because of its higher receiver sensitivity [1-3] and can profit from the advantage of integrated optics to realize stable and compact interferometers and balanced detectors. However, there are some impairments present in one bit- delay interferometers for balanced DPSK detection [3]: arising from amplitude imbalance, finite extinction ratio of the interferometer, phase imbalance, delay-tobit rate mismatch, frequency offset and polarisation dependent delay. Furthermore, the higher the data bit rate the more difficult it is to mitigate the aforementioned degradations.

We propose a novel DPSK receiver using Fibre Bragg Gratings (FBGs) which alleviates the stabilisation drawbacks of one-bit-delay balanced DPSK receivers. Two similar approaches have been proposed before $[4,5]$, but to the 
best of our knowledge, this is the first time that a correct recovery of the optical DPSK signal is demonstrated.

\section{SYSTEM DESIGN}

The phase shift keying modulation consists on encoding a binary data stream as phase shifts in a signal [6]. For an optical wave, this can be represented with the following expression:

$$
E(t)=\sqrt{P_{0}} e^{j\left(\omega_{0} t+\Delta \phi \sum_{k} a_{k} p\left(t-k T_{b}\right)\right)}
$$

where $P_{0}$ is the electrical field power (constant), $\omega_{0}$ is the electrical field central frequency, $a_{k}$ are the binary symbols, $p(t)$ is the electric pulse shape, $\Delta \phi$ is the phase shift corresponding to each binary transition, usually $\pi$ and $T_{b}$ the bit period. In particular, optical DPSK consists in encoding a logical change in the bit stream, represented by the summation term in Eq. 1 by a phase shift of the optical wave [6]. Hence, if we consider ideal rectangular pulses:

$$
p(t)=\prod\left(\frac{t-T_{b} / 2}{T_{b}}\right)
$$

the instantaneous frequency of the electrical field is given by:

$$
\begin{aligned}
f_{i} & =\frac{1}{2 \pi} \frac{\partial \phi(t)}{\partial t} \\
f_{i} & =f_{0}+\frac{1}{2} \sum_{k} a_{k}\left[\delta\left(t-k T_{b}\right)-\delta\left(t-(k+1) T_{b}\right)\right]
\end{aligned}
$$

Hence, the phase shift can be also regarded as an instantaneous frequency shift, so a data transition from 1 to 0 corresponds to an instantaneous down frequency shift, while a 0 to 1 transition results in an instantaneous up frequency shift, which is represented by the delta functions in Eq. 3. Therefore, all the information from the original bit stream is encoded in these instantaneous frequency shifts.

Based on this properties, we propose a novel receiver scheme to recover data using direct detection and optical pre-filtering. The setup for the receiver is shown in Fig. 1.

Thus, to detect properly the optical signals, two FBG cantered in the upper (FBG+) and lower (FBG-) frequencies with respect to the central wavelength can be placed as shown in Fig. 1. This splits the positive and negative frequency shifts, which after direct detection are transformed in intensity peaks. After the photodetectors some simple electronic components can be employed to convert the detected transitions in the received pulses. 


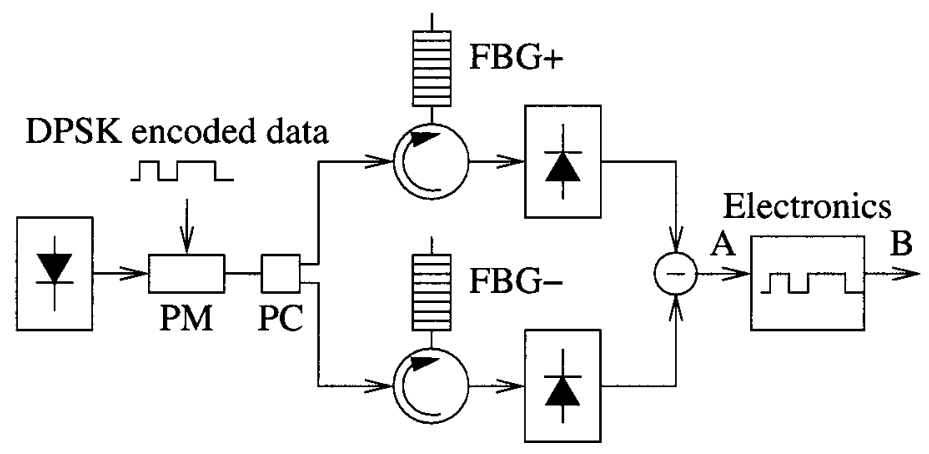

Figure 1. Schematic diagram of the proposed DPSK receiver(PM: Phase modulator, PC: Power Coupler, FBG: Fibre Bragg Grating).

\section{SIMULATIONS}

The receiver was simulated using available commercial software (VPItransmissionMaker), and the results are shown in Fig. 2, for a $10 \mathrm{Gbps}$ bit stream. The figure shows the intensity peaks corresponding to the transitions, detected each time data symbols change from 0 to 1 and from 1 to 0 (dark line), and how using a comparator and a low-pass filter, the bit stream is reconstructed (light line). The simulations were carried out for data rates from $2.5 \mathrm{Gbps}$ up to $40 \mathrm{Gbps}$, and in all the cases we found good agreement between the theory and the simulations.

\section{EXPERIMENTAL RESULTS}

We have experimentally implemented the set-up presented in Fig. 1, upto point $\mathrm{A}$, to test the theoretical analysis and simulations. Only a single branch (one FBG and a single optical photodetector, followed by and oscilloscope) was used, replacing the FBG conveniently to measure the positive and negative frequency shifts respectively. We used a continuous wave tunable laser source with an output optical power of $-2 \mathrm{dBm}$. An external optical phase modulator was driven by $10 \mathrm{~Gb} / \mathrm{s}$ PRBS sequence. The bit sequence length was $2^{7}-1$ with a mark probability for the logical " $1 "$ of $7 / 8$, in order to obtain a small number of logical " 0 " in the fixed pattern. The FBG had a reflectivity of 0.5 with a Full-Width Half Maximum of $6 \mathrm{GHz}$. The FBG was used in reflection, as depicted in Fig. 1.

The results are plotted in Fig. 3. In both plots, the electrical transmitted signal and the detected pulse edge transitions are shown, both for the negative and positive frequency shifts, from the laser nominal frequency. From the figure, there is a meaningful agreement between the experimental data and the results from the simulations shown in Fig. 2. 


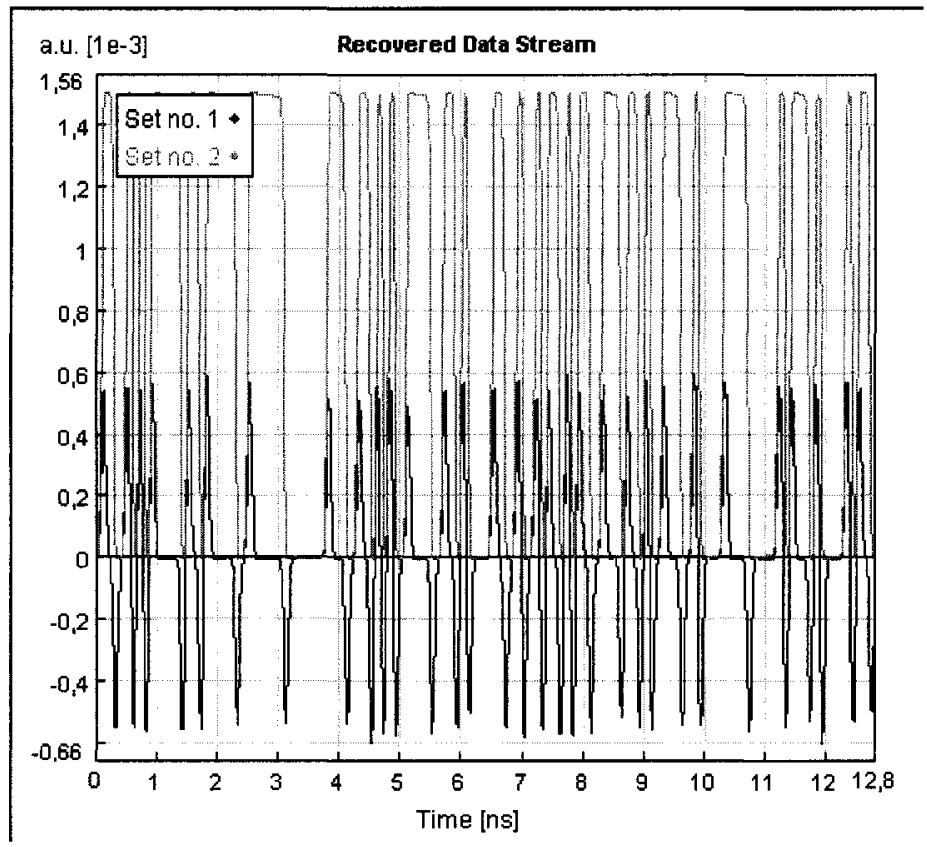

Figure 2. Simulation results intensity peaks at point A (dark line) of Fig. 1 and recovered bit stream (light line) at point B of Fig. 1.

The scale of the oscilloscope is $50 \mathrm{mV} / \mathrm{div}$ and $1 \mathrm{~ns} / \mathrm{div}$. The upper trace of each plot in Fig. 3 is the transmitted signals and the lower trace is the recovered data at point $A$ in the setup shown in Fig. 1. Fig. 3-(a) shows the detected pulse leading edges when the FBG is placed at frequencies lower than the optical carrier, which is in good agreement with the theory and simulations. Fig. 3-(b) shows the other case, with similar results and conclusions. Also, some ripples are observed in the figures, which are attributed to the fact that each time the spectrum is shifted some of the signal energy falls within the FBG side lobes, and is detected by the photodiode. This can be alleviated by a proper design in the pulse shaping electronics stage after detection, from A to B in Fig. 1.

Fig. 4 shows the results for the case when the FBG is aligned with the transmitter wavelength. It can be seen that each time a logical " 0 " (a transition in the upper trace) is transmitted, the detected power decreases due to the shift of the spectrum. Thus, the receiver scheme of Fig. 1 can be simplified to use a single FBG placed at the central wavelength at the expenses of receiver sensitivity, as has been also reported by other authors [5]. 


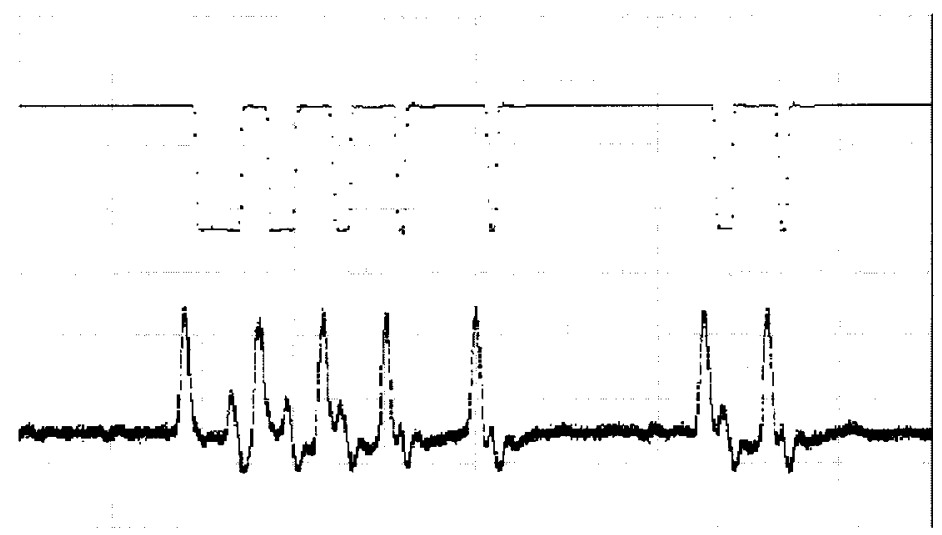

(a)

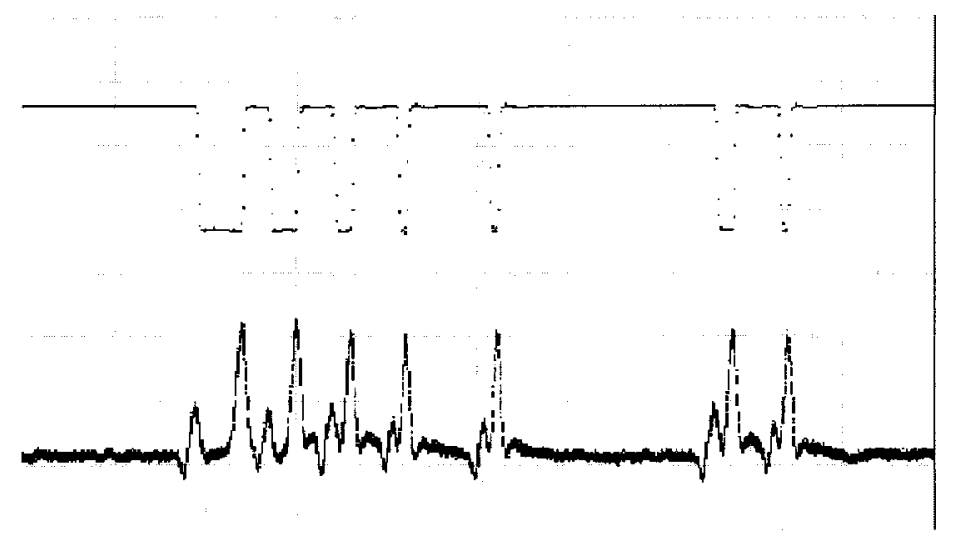

(b)

Figure 3. Transmitted electrical signal and measured pulse edge detection for (a) negative (FBG-) and (b) positive (FBG + ) frequency shifts.

\section{CONCLUSIONS}

We have proposed a novel receiver scheme for DPSK signals which alleviates the impairments of stabilisation of the conventional one-bit-delay optical interferometer. The receiver is based in optical separation, using a filter as for example a fibre Bragg grating, of the lower and upper frequency of a optical PSK signal, which detected independently, yielding intensity peaks after the photodiodes at instants corresponding to the trailing and leading edges, respectively, of the transmitted pulses. Although the FBG requires thermal stabilisation, it is less complex than stabilisation of optical interferometers. In 
the interferometer configuration, increasing the bit rate is challenging, since higher precision is needed in the delay line. Conversely, the proposed receiver is advantageous in this sense, since it becomes simpler to realize for operation at high bit-rates, because the slopes of the FBGs can then be smoother, and therefore easy to fabricate. Moreover, for optical interferometer DPSK demodulators operating at high bit rates, stabilisation issues become stricter. One aspect of concern of the proposed scheme is its efficiency in terms of detected energy; however, as it can be seen in Fig. 3 the signal-to-noise ratio of the detected signals is good enough for further signal processing. Techniques to improve the performance of the proposed scheme are under study such as pulse shaping and grating design.

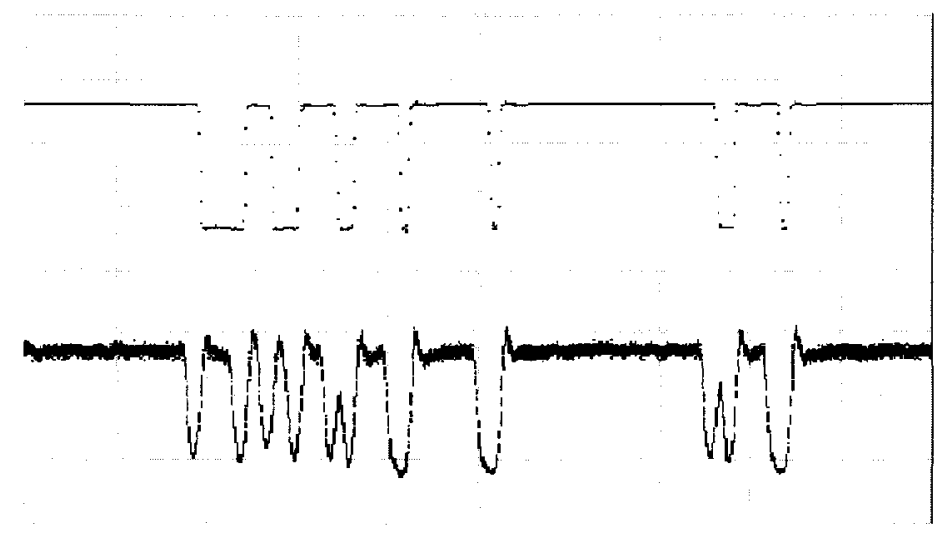

Figure 4. Detected signal when the FBG is aligned with the transmitter wavelength.

\section{REFERENCES}

[1] J. Sinsky, A. Adamiecki, A. Gnauck, C. Burrus, J. Leuthold, O. Wohlgemuth, S. Chandrasekhar, and A. Umbach, "RZ-DPSK transmission using a 42.7-Gb/s integrated balanced optical front end with record sensitivity," J. Lightwave Technol, vol. 22, pp. 180-185, Jan. 2004.

[2] D. Gill, A. Gnauck, X. Liu, X. Wei, and Y. Su, " $\pi / 2$ alternate-phase on-off keyed 42.7 $\mathrm{Gb} / \mathrm{s}$ long-haul transmission over $1980 \mathrm{~km}$ of standard single-mode fiber," IEEE Photon. Technol. Lett., vol. 16, pp. 906-908, Mar. 2004.

[3] P. Winzer and K. Hoon, "Degradations in balanced DPSK receivers," IEEE Photon. Technol. Lett., vol. 15, pp. 1282-1284, Sept. 2003.

[4] A. Royset and D. Hjelme, "Novel dispersion tolerant optical duobinary transmitter using phase modulator and bragg grating filter," in European Conference on Optical Communications, vol. 1, pp. 225 226, Sept. 1998.

[5] D. Penninckx, H. Bissessur, P. Brindel, E. Gohin, and F. Bakhti, "Optical differential phase shift keying (DPSK) direct detection considered as a duobinary signal," in European Conference on Optical Communications, vol. 3, pp. 456 457, September 2001.

[6] B. Sklar, Digital Communications. Prentice Hall, 2nd ed., 2001. 